\title{
Wavefronts and Light Cones for Kerr Spacetimes
}

\author{
Francisco Frutos-Alfaro ${ }^{1}$, Frank Grave ${ }^{2}$, Thomas Müller ${ }^{2}$, Daria Adis $^{3}$ \\ ${ }^{1}$ Department of Physics, University of Costa Rica, San Pedro, Costa Rica \\ ${ }^{2}$ Institute for Visualization und Interactive Systems, University of Stuttgart, Stuttgart, Germany \\ ${ }^{3}$ Theoretical Astrophysics, University of Tübingen, Tübingen, Germany \\ Email: frutos@fisica.ucr.ac.cr
}

Received September 26, 2012; revised October 27, 2012; accepted November 5, 2012

\begin{abstract}
We investigate the light propagation by means of simulations of wavefronts and light cones for Kerr spacetimes. Simulations of this kind give us a new insight to better understand the light propagation in presence of massive rotating black holes. A relevant result is that wavefronts are backscattered with winding around the black hole. To generate these visualizations, an interactive computer program with a graphical user interface, called JWFront, was written in Java.
\end{abstract}

Keywords: General Relativity; Wavefronts; Lightcones; Simulations

\section{Introduction}

In general relativity and astrophysics, Kerr spacetimes are useful to study, for example, stellar compact objects, like accretion disks in neutron stars. This metric was found by Kerr in 1963 [1], since then this spacetime appears in many articles on these topics and it is currently one of the most used metric, because this represents a spacetime of a massive rotating object.

Friedrich and Stewart [2,3] based on Arnold's catastrophe theory [4] developed the theory of wavefronts and singularities (caustics) in general relativity. Recently, Hasse [5] et al., Low [6], and Ehlers and Newman [7] have revived this topic from a mathematical viewpoint. The wavefront propagation, caustic and the light cone structures for a non rotating object, described with Schwarzschild spacetime, was discussed by Perlick [8]. Caustics for the Kerr metric were numerically computed by Rauch and Blandford [9]. Grave studied the gravitational collapse and wavefronts for this spacetime [10]. More recently, Sereno and De Luca [11] computed these caustics using a Taylor expansion of lightlike geodesics. A numerical treatment on the structure of Kerr caustics was done by Bozza [12]. Qualitative descriptions of wavefronts and caustics for gravitational lensing were presented by Blandford and Narayan [13], Schneider et al. [14], and Ohanian and Ruffini [15]. Petters et al. [16,17], and Frittelli and Petters [18] addressed formally this subject. Ellis et al. [19] discussed qualitatively the light cone structure for gravitational lensing.

In this work, wavefronts, caustics and light cones for the Kerr spacetime are investigated. The best way to tackle it is through computer simulations. Nowadays, these simulations are becoming relevant in general relativity, because they can help understand complex phenomena. With the new technologies, these simulations can practically be done in real time. Thus, the aim of this work is to provide a new perspective about wavefront propagations in Kerr spacetimes by means of computer simulation. For this purpose, we have designed JWFront [20], an interactive Java program using OpenGL (Open Graphics Library), to visualize wavefronts and light cones for this spacetime.

In the next section, the Kerr spacetime and its tetrads will be briefly introduced and discussed. The equation of motion, i.e. the geodesic equation and how it is solved, will be discussed in the third section. Definitions for the sake of visualizations about the wavefront, caustic and light cone structures are presented in the fourth section. A succinct discussion about our program JWFront will be given in the fifth section. The last section is devoted to discuss the results of the visualizations for the Kerr spacetime. From these simulations, one can see the evolution of wavefronts and light cones providing new perspectives for understanding them.

\section{Kerr Spacetimes}

\subsection{Kerr Metric}

The Kerr metric is an exact solution of the vacuum Einstein field equation and represents the spacetime of a massive rotating black hole. In this spacetime, the rotating body would exhibit an inertial frame dragging (Lense-Thirring effect), i.e., a particle moving close to it would corotate. This is not because of any force or torque applied on the particle, but rather because of the space- 
time curvature associated with this black hole. This region is called the ergosphere. At large distances this spacetime is flat (asymptotically flat). In Boyer-Linquist coordinates the metric has the following form $[21,22]$ :

$$
\begin{aligned}
\mathrm{d} s^{2}= & -\frac{\Delta}{\rho^{2}}\left[\mathrm{~d} t-a \sin ^{2} \theta \mathrm{d} \varphi\right]^{2}+\frac{\Delta}{\rho^{2}} \mathrm{~d} r^{2}+\rho^{2} \mathrm{~d} \theta^{2} \\
& +\frac{\sin ^{2} \theta}{\rho^{2}}\left[\left(r^{2}+a^{2}\right) \mathrm{d} \varphi-a \mathrm{~d} t\right]^{2} \\
= & g_{00} \mathrm{~d} t^{2}+g_{03} \mathrm{~d} t \mathrm{~d} \varphi+g_{11} \mathrm{~d} r^{2}+g_{22} \mathrm{~d} \theta^{2}+g_{33} \mathrm{~d} \varphi^{2},
\end{aligned}
$$

where

$$
\begin{aligned}
& \Delta=r^{2}-R_{S} r+a^{2}, \\
& \rho^{2}=r^{2}+a^{2} \cos ^{2} \theta,
\end{aligned}
$$

$R_{S}$ is the Schwarzschild radius in geometrical units ( $c=G=1$ ), $M$ is the mass of the black hole, $a=J / M$ (angular momentum per unit mass, $a \leq M$ ), and $g_{\mu v}$ are the metric components, which can be read off easily from (1). The Kerr spacetime contains the Minkowski flat metric, the Schwarzschild metric, and the LenseThirring spacetime. If $a^{2} \rightarrow 0$, i.e. neglecting the second order in powers of $a$, one gets the Lense-Thirring metric, which represents the metric of a massive slowrotating body. We get the Schwarzschild metric if $a=0$, which represents the metric of a massive non-rotating body.

\subsection{Local Frames: Tetrad Formalism}

The tetrad formalism is very useful in general relativity. It defines a mathematical element called tetrad or vierbein, which is used to connect the curved coordinate systems with the local flat Lorentz coordinates. These tetrads must fulfill the equation

$$
\eta_{(\mu)(v)}=g_{\alpha \beta} e_{(\mu)}^{\alpha} e_{(v)}^{\beta},
$$

where $e_{(\mu)}^{\alpha}$ is a chosen vierbein element, $\eta_{(\mu)(v)}$ stands for the Minkowski metric (diag $(-1,1,1,1))$.

For the Kerr spacetime, there are at least two possibilities to choose these tetrads. The first one is called the locally static frame (LSF). In this frame, the observer is static. This kind of observer cannot be located in the ergosphere, because they would move with superluminal velocity in this region to counteract the Lense-Thirring effect. The local tetrads for this static observer have following components:

$$
\begin{aligned}
& e_{(0)}=\frac{1}{\sqrt{-g_{00}}} \frac{\partial}{\partial t}=\frac{\rho}{\sqrt{\rho^{2}-R_{s} r}} \frac{\partial}{\partial t} \\
& e_{(1)}=\frac{1}{\sqrt{g_{11}}} \frac{\partial}{\partial r}=\frac{\sqrt{\Delta}}{\rho} \frac{\partial}{\partial r}
\end{aligned}
$$

$$
\begin{aligned}
e_{(2)} & =\frac{1}{\sqrt{g_{22}}} \frac{\partial}{\partial \theta}=\frac{1}{\rho} \frac{\partial}{\partial \theta} \\
e_{(3)} & =-\frac{g_{03}}{\sqrt{g_{00}\left(g_{00} g_{33}-g_{03}^{2}\right)}} \frac{\partial}{\partial t}+\sqrt{\frac{g_{00}}{g_{00} g_{33}-g_{03}^{2}}} \frac{\partial}{\partial \varphi} \\
& =\frac{R_{S} \operatorname{ar} \sin \theta}{\rho \sqrt{\Delta\left(\rho^{2}-R_{S} r\right)}} \frac{\partial}{\partial t}+\frac{1}{\rho \sin \theta} \sqrt{\frac{\rho^{2}-R_{S} r}{\Delta}} \frac{\partial}{\partial \varphi},
\end{aligned}
$$

where $\partial / \partial t, \partial / \partial r, \partial / \partial \theta$ and $\partial / \partial \varphi$ are understood as unit vector directions.

The second one is called locally nonrotating frame [23] (LNRF), in which the observer is stationary. An observer in this kind of frames could be in the ergosphere. The local tetrads for this stationary observer have following components:

$$
\begin{aligned}
e_{(0)} & =\sqrt{\frac{g_{33}}{g_{03}^{2}-g_{00} g_{33}}} \frac{\partial}{\partial t}-\frac{g_{03}}{\sqrt{g_{33}\left(g_{03}^{2}-g_{00} g_{33}\right)}} \frac{\partial}{\partial \varphi} \\
& =\frac{\Sigma}{\rho \sqrt{\Delta}} \frac{\partial}{\partial t}+\frac{R_{S} a r}{\rho \Sigma \sqrt{\Delta}} \frac{\partial}{\partial \varphi} \\
e_{(1)} & =\frac{1}{\sqrt{g_{11}}} \frac{\partial}{\partial r}=\frac{\sqrt{\Delta}}{\rho} \frac{\partial}{\partial r} \\
e_{(2)} & =\frac{1}{\sqrt{g_{22}}} \frac{\partial}{\partial \theta}=\frac{1}{\rho} \frac{\partial}{\partial \theta} \\
e_{(3)} & =\frac{1}{\sqrt{g_{33}}} \frac{\partial}{\partial \varphi}=\frac{\rho}{\Sigma \sin \theta} \frac{\partial}{\partial \varphi},
\end{aligned}
$$

where $\Sigma=\left(r^{2}+a^{2}\right)^{2}-a^{2} \Delta \sin ^{2} \theta$. These tetrad definitions are useful to find the trajectories of light rays moving in a Kerr spacetime.

\section{The Geodesic Equation and Its Solution}

\subsection{Geodesic Equation}

In general relativity, the trajectory of particles or light rays can be determined by the geodesic equation. Generally, this equation can only be solved using numerical methods. This equation has the following form [21,22]:

$$
\frac{\mathrm{d}^{2} x^{\mu}}{\mathrm{d} \lambda^{2}}+\Gamma_{\alpha \beta}^{\mu} \frac{\mathrm{d} x^{\alpha}}{\mathrm{d} \lambda} \frac{\mathrm{d} x^{\beta}}{\mathrm{d} \lambda}=0,
$$

where $\alpha, \beta, \mu=0,1,2,3$, and $\lambda$ is an affine parameter, a parameter such that $\mathrm{d} x^{\beta} / \mathrm{d} \lambda$ has constant magnitude (affine parametrization). The components $\Gamma_{\beta \gamma}^{\alpha}$, called the Christoffel symbols, are given by

$$
\Gamma_{\alpha \beta}^{\mu}=\frac{g^{\mu v}}{2}\left[\frac{\partial g_{\alpha v}}{\partial x^{\beta}}+\frac{\partial g_{\beta v}}{\partial x^{\alpha}}-\frac{\partial g_{\alpha \beta}}{\partial x^{v}}\right] .
$$

These symbols for the Kerr metric can be computed by means of symbolic programs. A program using the free 
symbolic software REDUCE [24] was written to obtain them. In the Appendix, the non-null Christoffel symbols are listed. Introducing these Christoffel symbols into Equation (5), one has four ordinary second order differential equations given by:

$$
\begin{aligned}
& \frac{\mathrm{d}^{2} t}{\mathrm{~d} \lambda^{2}}+2 \frac{\mathrm{d} t}{\mathrm{~d} \lambda}\left(\Gamma_{01}^{0} \frac{\mathrm{d} r}{\mathrm{~d} \lambda}+\Gamma_{02}^{0} \frac{\mathrm{d} \theta}{\mathrm{d} \lambda}\right) \\
& +2 \frac{\mathrm{d} \varphi}{\mathrm{d} \lambda}\left(\Gamma_{13}^{0} \frac{\mathrm{d} r}{\mathrm{~d} \lambda}+\Gamma_{23}^{0} \frac{\mathrm{d} \theta}{\mathrm{d} \lambda}\right)=0, \\
& \frac{\mathrm{d}^{2} r}{\mathrm{~d} \lambda^{2}}+\Gamma_{00}^{1}\left(\frac{\mathrm{d} t}{\mathrm{~d} \lambda}\right)^{2}+2 \Gamma_{03}^{1} \frac{\mathrm{d} t}{\mathrm{~d} \lambda} \frac{\mathrm{d} \varphi}{\mathrm{d} \lambda}+\Gamma_{11}^{1}\left(\frac{\mathrm{d} r}{\mathrm{~d} \lambda}\right)^{2} \\
& +2 \Gamma_{12}^{1} \frac{\mathrm{d} r}{\mathrm{~d} \lambda} \frac{\mathrm{d} \theta}{\mathrm{d} \lambda}+\Gamma_{22}^{1}\left(\frac{\mathrm{d} \theta}{\mathrm{d} \lambda}\right)^{2}+\Gamma_{33}^{1}\left(\frac{\mathrm{d} \varphi}{\mathrm{d} \lambda}\right)^{2}=0, \\
& \frac{\mathrm{d}^{2} \theta}{\mathrm{d} \lambda^{2}}+\Gamma_{00}^{2}\left(\frac{\mathrm{d} t}{\mathrm{~d} \lambda}\right)^{2}+2 \Gamma_{03}^{2} \frac{\mathrm{d} t}{\mathrm{~d} \lambda} \frac{\mathrm{d} \varphi}{\mathrm{d} \lambda}+\Gamma_{11}^{2}\left(\frac{\mathrm{d} r}{\mathrm{~d} \lambda}\right)^{2} \\
& +2 \Gamma_{12}^{2} \frac{\mathrm{d} r}{\mathrm{~d} \lambda} \frac{\mathrm{d} \theta}{\mathrm{d} \lambda}+\Gamma_{22}^{2}\left(\frac{\mathrm{d} \theta}{\mathrm{d} \lambda}\right)^{2}+\Gamma_{33}^{2}\left(\frac{\mathrm{d} \varphi}{\mathrm{d} \lambda}\right)^{2}=0, \\
& \frac{\mathrm{d}^{2} \varphi}{\mathrm{d} \lambda^{2}}+2 \frac{\mathrm{d} t}{\mathrm{~d} \lambda}\left(\Gamma_{01}^{3} \frac{\mathrm{d} r}{\mathrm{~d} \lambda}+\Gamma_{02}^{3} \frac{\mathrm{d} \theta}{\mathrm{d} \lambda}\right) \\
& +2 \frac{\mathrm{d} \varphi}{\mathrm{d} \lambda}\left(\Gamma_{13}^{3} \frac{\mathrm{d} r}{\mathrm{~d} \lambda}+\Gamma_{23}^{3} \frac{\mathrm{d} \theta}{\mathrm{d} \lambda}\right)=0 .
\end{aligned}
$$

For light rays, there is also another equation they have to fulfill, the null geodesic equation (lightlike geodesics):

$$
\begin{aligned}
\left(\frac{\mathrm{d} s}{\mathrm{~d} \lambda}\right)^{2} & =g_{\mu \nu} \frac{\mathrm{d} x^{\mu}}{\mathrm{d} \lambda} \frac{\mathrm{d} x^{v}}{\mathrm{~d} \lambda} \\
& =g_{00}\left(\frac{\mathrm{d} t}{\mathrm{~d} \lambda}\right)^{2}+2 g_{03}\left(\frac{\mathrm{d} t}{\mathrm{~d} \lambda}\right)\left(\frac{\mathrm{d} \varphi}{\mathrm{d} \lambda}\right) \\
& +g_{11}\left(\frac{\mathrm{d} r}{\mathrm{~d} \lambda}\right)^{2}+g_{22}\left(\frac{\mathrm{d} \theta}{\mathrm{d} \lambda}\right)^{2}+g_{33}\left(\frac{\mathrm{d} \varphi}{\mathrm{d} \lambda}\right)^{2}=0 .
\end{aligned}
$$

The four-dimensional trajectories of light rays can be found by solving the Equations (6) with the constraint Equation (7). Now, we need initial conditions in order to solve these equations numerically.

\subsection{Initial Conditions}

The initial spacetime event $x_{0}^{\mu}=\left(t_{0}, x_{0}, y_{0}, z_{0}\right)$ for all geodesics of the bundle defining the wavefront (see below) has to be given in order to solve numerically Equations (6) with the constraint Equation (7). For each geodesic of the bundle, the four-velocity at the initial point,

$$
\left[\frac{\mathrm{d} x^{\mu}}{\mathrm{d} \lambda}\right]_{0}=\left(\frac{\mathrm{d} t}{\mathrm{~d} \lambda}, \frac{\mathrm{d} x}{\mathrm{~d} \lambda}, \frac{\mathrm{d} y}{\mathrm{~d} \lambda}, \frac{\mathrm{d} z}{\mathrm{~d} \lambda}\right)_{0},
$$

determines the direction for each geodesic and they are calculated as follows: the tridimensional (3D) initial vec- tor,

$$
\left[\frac{\mathrm{d} \tilde{x}}{\mathrm{~d} \lambda}\right]_{0}=\left(\frac{\mathrm{d} \tilde{x}}{\mathrm{~d} \lambda}, \frac{\mathrm{d} \tilde{y}}{\mathrm{~d} \lambda}, \frac{\mathrm{d} \tilde{z}}{\mathrm{~d} \lambda}\right)_{0},
$$

for each geodesic in local flat spacetime is given input. Using the null geodesic condition for this local metric, the initial time derivative $[\mathrm{d} \tilde{t} / \mathrm{d} \lambda]$ is determined. Now, we have all components in local flat spacetime $\left[\mathrm{d} \tilde{x}^{\mu} / \mathrm{d} \lambda\right]_{0}$. Finally, the four-velocity in non-flat spacetime is determined by transforming from the local flat spacetime to the non-flat spacetime using the tetrads $e_{(v)}^{\mu}$ for the Kerr metric:

$$
\left[\frac{\mathrm{d} x^{\mu}}{\mathrm{d} \lambda}\right]_{0}=e_{(v)}^{\mu}\left[\frac{\mathrm{d} \tilde{x}^{v}}{\mathrm{~d} \lambda}\right]_{0}
$$

With the purpose of simulating wavefronts and light cones in mind, one has to choose between the two kinds of observers (see Section 2).

Now, we have all elements to numerically solve the four ordinary equations with these initial conditions. For this goal, a fourth order Runge-Kutta procedure is used.

\section{Wavefronts, Caustics and Light Cones}

\subsection{Wavefronts}

Formally speaking, the wavefronts are defined as follows: A wavefront is generated by a bundle of light rays orthogonal to a spacelike 2-surface in a four-dimensional Lorentzian manifold [5].

To simulate it, the wavefront is defined as the surface $A$ generated by all points of the null geodesic bundle at a given time:

$$
A\left(t_{i}\right)=\left\{\begin{array}{c}
\gamma\left(t_{i}\right) \text { is a null geodesic with } \\
\gamma\left(t_{0}\right)=\left(t_{0}, x_{0}, y_{0}, z_{0}\right), \quad t_{i} \geq t_{0}
\end{array}\right\} .
$$

Qualitatively speaking, the wavefronts that spread out in all directions from the source are spherical at the very beginning and if they are approaching a deflector, they get distorted and their sheets develop generally singularities: cusp ridges, self intersections and caustics.

In gravitational lens theory, it is considered that light ray deflection occurs only at the place where the deflector is located (thin lens approximation). This approximation is very useful in many calculations, specially, if we are dealing with strong lensing. Under this consideration, wavefronts propagate spherically without any perturbation until the deflector, then wavefronts are distorted by the deflector. The general case is completely different, because wavefronts get already perturbed before they approach the deflector and can wind around the black hole (see Figure 1). An observer which is behind the deflector will see different sheets of the same wavefront coming from different directions. Then, the observer will 

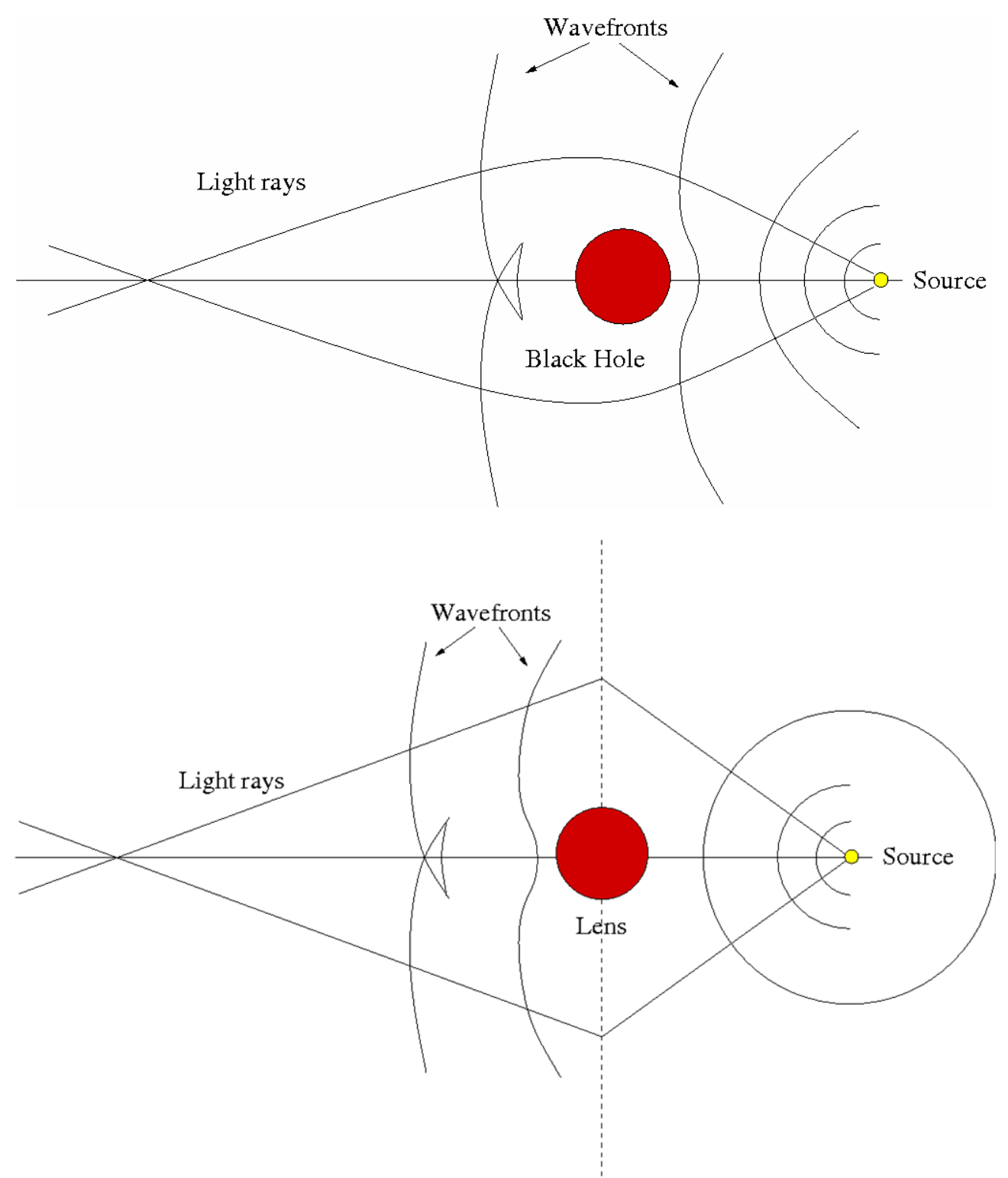

Figure 1. Differences in the evolution of wavefront in presence of a black hole (top) and a gravitational lens (bottom).

think that there are multiple images of the same source.

\subsection{Caustics}

A caustic of a wavefront is formally defined as the set of all points where the wavefront fails to be an (immersed) submanifold [5].

Roughly speaking, a caustic is the envelope of reflected or refracted light rays by a curved surface or object. A caustic can be a point, a line or a surface. For instance, for the Schwarzschild black hole the caustic is a line along of the line of sight, and for the point mass lens or non-rotating black hole the caustic is a point in the line of sight. Interesting caustic shapes can be found in gravitational lens theory, for example, for some elliptical lens models, it is common to find diamond shape caustics. Another important point to mention about caustics is that if an observer would be on a caustic, he would detect a high light intensity (mathematically speaking, it would be infinity).

\subsection{Light Cones}

The light cone is defined as the surface generated by all points $(t, x, y, z)$, that fulfill the geodesic equation with the null geodesic condition for a fixed starting event $x_{0}^{\mu}=\left(t_{0}, x_{0}, y_{0}, z_{0}\right)$. To visualize the light cones, one has to suppress one space dimension, using for instance the coordinates $(t, x, y),(t, x, z)$ or $(t, y, z)$. Light cones can also be used to visualize caustic structures [13], because 
time slices or cuts in the light cones represent the development of the wavefront. The same differences that appeared in the structures of wavefronts are also expected in light cones.

In the present work, we will mainly concentrate on visualizations of wavefronts and light cones. For more mathematical details about wavefronts, caustics and light cones, the interested reader may consult the references at the end. Details of the simulations will be shown in the sixth section.

\section{JWFront}

An interactive frontend or GUI (graphical user interface) to visualize wavefronts and light cones in general relativity, called JWFront, was written in Java [20]. Basically, on this GUI, the user have to enter the initial position values and choose the values for mass and angular momentum per unit mass ( $M$ and $a$ ). Later, the user can choose what to see. Among the applications, the user can get from our program, are:
- Wavefront animations in 2D and 3D,

- Light cone visualizations.

The light cones are visualized using the coordinate systems $(t, x, y)$ or $(t, z, x)$. All data obtained from solving the equations is processed in the program by means of Java and OpenGL subroutines in order to simulate wavefronts and light cones.

Moreover, this Java program can be easily modified to simulate wavefronts and light cones for other spacetime. The user just has to provide the Christoffel symbols into the program.

The interested reader may send us a message requesting for the program or for more information about it.

\section{Simulation with JWFront}

Now, let us discuss some examples of the simulations obtained by JWFront (see Figures 2-4). Figures 2-4 are visualizations for the Kerr spacetime with $M=1$ and $a=$ 0.9 .

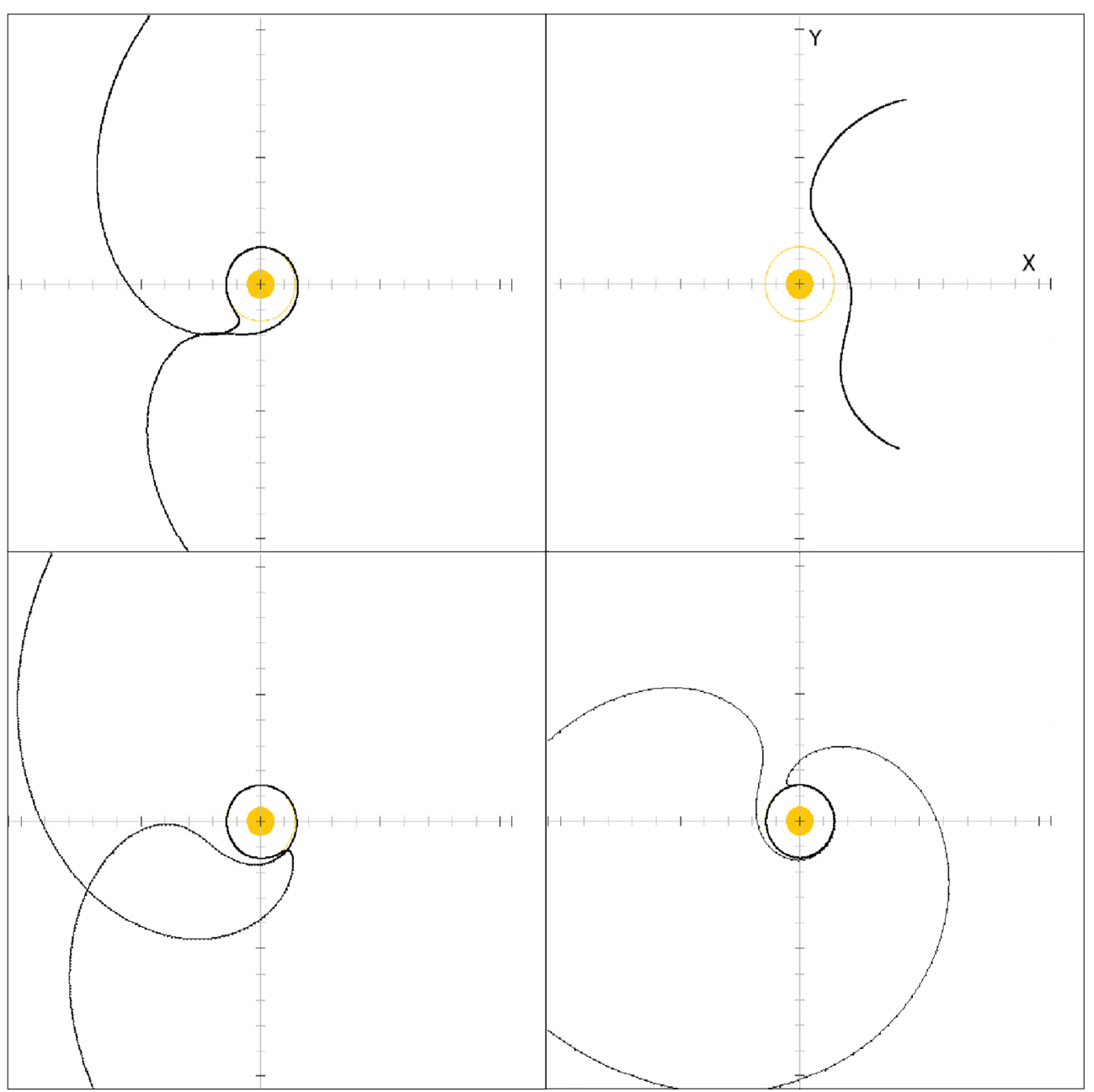

Figure 2. Two-dimensional wavefront sequence for the Kerr metric $(M=1, a=0.9)$. The sequence begins on the top left frame. The wavefront is moving from the right to the left in the $x y$ plane. 


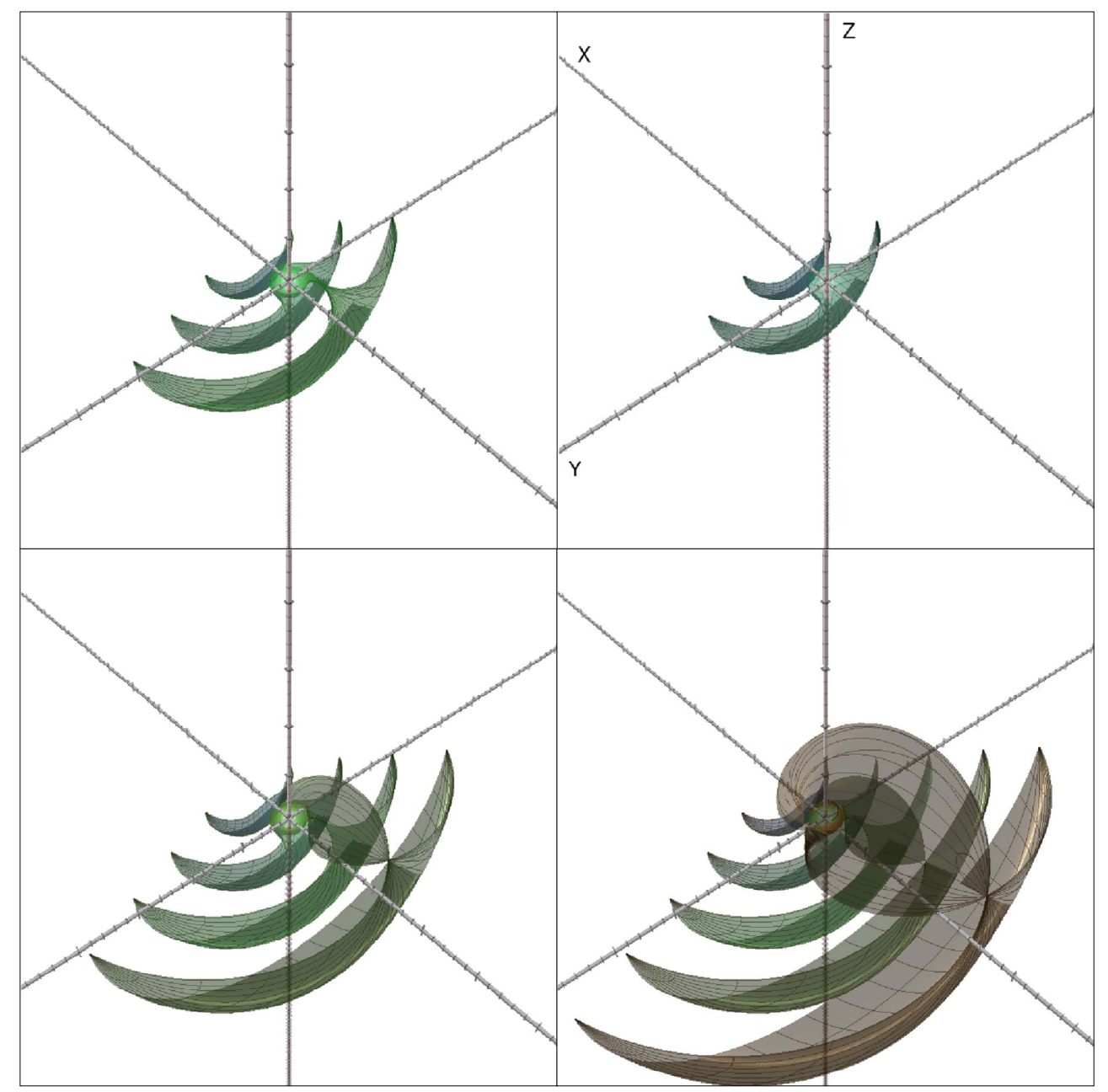

Figure 3. Three-dimensional wavefront sequence for the Kerr metric $(M=1, a=0.9)$. The sequence begins on the top left frame.

The 2D visualization of a wavefront (light pulse) moving from right to left is shown in Figure 2. In these frames, the inner horizon is displayed as a small filled circle, the ergo-region as a bigger circle. Because of the rapidly rotation of the black hole, the wavefront is not symmetric in this plane. The black hole rotates counterclockwise, and so that the upper part of the wavefront reaches the $y$ axis earlier than the lower part. Furthermore, because the wavefront infinitely winds around the black hole from left to right and right to left, the observer will not see a continuously visible Einstein ring as in the case of a nonrotating black hole. An observer located in the intersection point of the wavefront with itself can see the initial light pulse coming from two direction in this plane.

In Figure 3, the 3D visualizations of a wavefront are shown. In this Figure, the wavefront consists of $1 / 8$ of a sphere defined by the initial local directions. Certain steps of the wavefront motion are included in every frame. We can see that the wavefront, starting from below the $z$ axis, reaches positive $z$ values, because of the above winding effect. As explained with the last figures, every point of the spacetime (excluding those inside of the black hole) is reached by this wavefront. The visualizations of the light cones are shown in Figure $4((t, x$, $y$ ) coordinates). The structures observed in these frames are similar to the corresponding structures of Figure 2.

\section{Conclusion}

The simulations produced by JWFront helps understand the light propagation in strong gravitational fields with rotation, such as in Kerr spacetimes. An interesting feature of wavefronts propagation appeared: the wavefronts are backscattered and wind around the black hole. Thus, an observer on the line of sight with the deflector and the source would see multiple images, and if the black hole does not rotate, the observer would see at least one Einstein ring, if he or she is aligned with the black hole. For Schwarzschild metric this winding effect is symmetric whereas for the Kerr one it is not, this is due to black hole rotation. JWFront can also displayed the visualiza- 


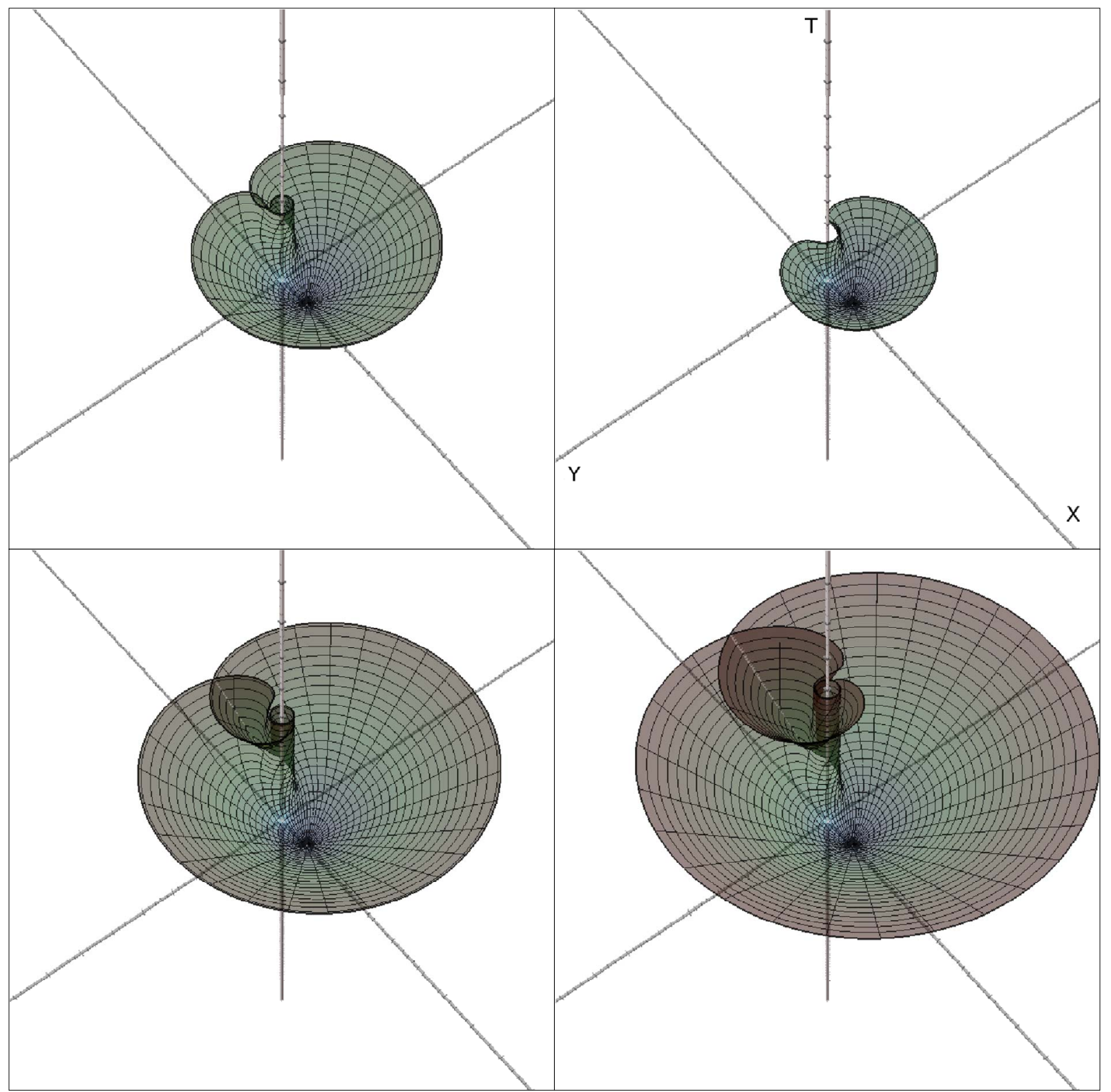

Figure 4. Light cone evolution for the Kerr metric $(M=1, a=0.9)$. The sequence begins on the top left frame. The light cone evolves from the initial point on the xyt space.

tions of light cones in these spacetimes. The results of the wavefront visualizations showed that the same structures can also be seen with light cone simulations as expected.

\section{Acknowledgements}

F. Frutos-Alfaro would like to thank Dr. rer.nat. Antonio Banichevich and Ph.D. Herberth-Morales for fruitful discussions.

\section{REFERENCES}

[1] R. P. Kerr, "Gravitational Field of a Spinning Mass as an Example of Algebraically Special Metrics,” Physical Re- view Letters, Vol. 11, No. 5, 1963, p. 237. doi:10.1103/PhysRevLett.11.237

[2] H. Friedrich and J. M. Stewart, "Characteristic Initial Data and Wavefront Singularities in General Relativity,” Proceedings of the Royal Society London, Series A, Vol. 385, No. 1789, 1983, pp. 345-371.

[3] J. M. Stewart, "Advanced General Relativity," Cambridge Monographs on Mathematical Physics, Cambridge University, Cambridge, 1993.

[4] V. I. Arnold, "Singularities of Caustics and Wavefronts," Kluwer, Amsterdam, 1990. doi:10.1007/978-94-011-3330-2

[5] W. Hasse, M. Kriele and V. Perlick, "Caustics of Wavefronts in General Relativity,” Classical and Quantum Grav- 
ity, Vol. 13, No. 5, 1996, pp. 1161-1182. doi:10.1088/0264-9381/13/5/027

[6] R. Low, "Stable Singularities of Wavefronts in General Relativity,” Journal of Mathematical Physics, Vol. 36, No. 8, 1998, pp. 3332-3335. doi:10.1063/1.532257

[7] J. Ehlers and E. Newman, "The Theory of Caustics and Wavefront Singularities with Physical Applications," Journal of Mathematical Physics, Vol. 41, 2000, pp. 33443378, arXiv:gr-qc/9906065. doi:10.1063/1.533316

[8] V. Perlick, "Gravitational Lensing from a Spacetime Perspective," Living Reviews in Relativity, Vol. 7, No. 9, 2004, arXiv:1010.3416.

[9] K. P. Rauch and R. Blandford, "Optical Caustics in a Kerr Spacetime and the Origin of Rapid X-Ray Variability in Active Galactic Nuclei,” The Astrophysical Journal, Vol. 421, No. 1, 1994, pp. 46-68. doi:10.1086/173625

[10] F. Grave, "Visualization of Gravitational Collapse and Wavefronts in the General Relativity Theory, Diplomarbeit,” Master Thesis, Eberhard Karls Universität Tübingen, Tübingen, 2004.

[11] M. Sereno and F. De Luca, "Primary Caustics and Critical Points behind a Kerr Black Hole," Physical Review D, Vol. 78, No. 2, 2008, Article ID: 023008, arXiv: astro$\mathrm{ph} / 0710.5923$.

[12] V. Bozza, "Optical Caustics of Kerr Spacetime: The Full Structure,” Physical Review D, Vol. 78, No. 6, 2008, Article ID: 063014, arXiv:gr-qc/0806.4102.

[13] R. D. Blandford and R. Narayan, "Cosmological Applications of Gravitational Lensing," Annual Review of Astronomy and Astrophysics, Vol. 30, 1992, pp. 311-358. doi:10.1146/annurev.aa.30.090192.001523

[14] P. Schneider, J. Ehlers and E. E. Falco, "Gravitational Lenses,” Springer, Berlin, 1992. doi:10.1007/978-1-4612-2756-4

[15] H. Ohanian and R. Ruffini, "Gravitation and Spacetime," W. W. Norton \& Company, New York, 1994.

[16] A. O. Petters, "Arnold's Singularity Theory and Gravitational Lensing," Journal of Mathematical Physics, Vol.
34, No. 8, 1993, pp. 3555-3581. doi:10.1063/1.530045

[17] A. O. Petters, H. Levine and J. Wambsganss, "Singularity Theory and Gravitational Lensing,” Springer, Berlin, 2001. doi:10.1007/978-1-4612-0145-8

[18] S. Frittelli and A. O. Petters. "Wave Fronts, Caustic Sheets, and Caustic Surfing in Gravitational Lensing," Journal of Mathematical Physics, Vol. 43, No. 11, 2002, pp. 5578-5611, arXiv:astro-ph/0208135. doi:10.1063/1.1511790

[19] G. F. R. Ellis, B. A. Bassett and P. K. S. Dunsby, "Lensing and Caustic Effects on Cosmological Distances," Classical and Quantum Gravity, Vol. 15, No. 8, 1998, pp. 2345-2361, arXiv:gr-qc/9801092v1. doi:10.1088/0264-9381/15/8/015

[20] F. Grave, F. Frutos-Alfaro, T. Müller and D. Adis, “JWFront in the Eleventh Marcel Grossmann Meeting: On Recent Developments in Theoretical and Experimental General Relativity,” In: H. Kleinert and R. T. Jantzen, Eds., Gravitation and Relativistic Field Theories, Proceedings of the MG11 Meeting, World Scientific Publishing Company, London, 2008.

[21] R. d’Inverno, “Introducing Einstein’s Relativity,” Oxford University Press, Oxford, 1992.

[22] C. W. Misner, K. S. Thorne and J. A. Wheeler, "Gravitation,” Freeman, San Francisco, 1973.

[23] J. M. Bardeen, W. H. Press and S. A. Teukolsky, "Rotating Black Holes: Locally Nonrotating Frames, Energy Extraction, and Scalar Synchrotron Radiation," The Astrophysical Journal, Vol. 178, 1972, pp. 347-369. doi:10.1086/151796

[24] A. C. Hearn, "REDUCE (User's and Contributed Packages Manual)," Konrad-Zuse-Zentrum für Informationstechnik, Berlin, 1999.

[25] O. Semerák, "Spinning Test Particles in a Kerr Field-I," Monthly Notices of Royal Astronomical Society, Vol. 308, No. 3, 1999, pp. 863-875. doi:10.1046/j.1365-8711.1999.02754.x 


\section{Appendix: Christoffel Symbols}

The non-zero Christoffel symbols for the Kerr metric are given by

$$
\begin{aligned}
& \Gamma_{01}^{0}=\frac{R_{s}}{2 \rho^{4} \Delta}\left(r^{2}+a^{2}\right)\left(2 r^{2}-\rho^{2}\right) \\
& \Gamma_{02}^{0}=\frac{-2 a J r}{\rho^{4}} \sin \theta \cos \theta \\
& \Gamma_{13}^{0}=\frac{-J \sin ^{2} \theta}{\rho^{4} \Delta}\left[\rho^{2}\left(r^{2}-a^{2}\right)+2 r^{2}\left(r^{2}+a^{2}\right)\right] \\
& \Gamma_{23}^{0}=\frac{2 a^{2} J r}{\rho^{4}} \cos \theta \sin ^{3} \theta \\
& \Gamma_{00}^{1}=\frac{R_{s} \Delta}{2 \rho^{6}}\left[2 r^{2}-\rho^{2}\right] \\
& \Gamma_{03}^{1}=\frac{-J \Delta}{\rho^{6}}\left(2 r^{2}-\rho^{2}\right) \sin ^{2} \theta \\
& \Gamma_{11}^{1}=\frac{1}{\rho^{2} \Delta}\left[\rho^{2}\left(\frac{R_{s}}{2}-r\right)+r \Delta\right] \\
& \Gamma_{33}^{1}=\frac{-\Delta \sin ^{2} \theta}{\rho^{6}}\left[r \rho^{4}-a J\left(2 r^{2}-\rho^{2}\right) \sin ^{2} \theta\right] \\
& \Gamma_{12}^{1}=\frac{-a^{2}}{\rho^{2}} \sin \theta \cos \theta \\
& \rho^{2}
\end{aligned}
$$

$$
\begin{aligned}
& \Gamma_{00}^{2}=\frac{-2 a J r}{\rho^{6}} \sin \theta \cos \theta \\
& \Gamma_{03}^{2}=\frac{2 J r}{\rho^{6}}\left(r^{2}+a^{2}\right) \sin \theta \cos \theta \\
& \Gamma_{11}^{2}=\frac{a^{2}}{\rho^{2} \Delta} \sin \theta \cos \theta \\
& \Gamma_{12}^{2}=\frac{r}{\rho^{2}} \\
& \Gamma_{22}^{2}=\Gamma_{12}^{1} \\
& \Gamma_{33}^{2}=\frac{-\sin \theta \cos \theta}{\rho^{6}}\left[\rho^{4} \Delta+R_{s} r\left(r^{2}+a^{2}\right)^{2}\right] \\
& \Gamma_{01}^{3}=\frac{J}{\rho^{4} \Delta}\left[2 r^{2}-\rho^{2}\right] \\
& \Gamma_{02}^{3}=\frac{-2 J r \cos \theta}{\rho^{4} \sin \theta} \\
& \Gamma_{13}^{3}=\frac{1}{\rho^{4} \Delta}\left[r \rho^{2}\left(\rho^{2}-R_{s} r\right)-a J \sin ^{2} \theta\left(2 r^{2}-\rho^{2}\right)\right] \\
& \Gamma_{23}^{3}=\frac{\cos \theta}{\rho^{4} \sin \theta}\left[\rho^{4}+2 a J r \sin ^{2} \theta\right]
\end{aligned}
$$

These Christoffel symbols coincide with the ones obtained by Smerák [25]. 